\title{
A New PID Tuning Method Based on Transient Response Control
}

\author{
S. E. Hamamci
}

\begin{abstract}
In this paper, an efficient PID tuning method for stable, unstable and integrating systems with time delay is introduced. The presented method is based on shaping the transient response of the two-degrees of freedom closed-loop system according to the performance specifications determined before the design. This method gives explicit tuning formulae in terms of plant model parameters and two design parameters which are used to shape the transient response. A graphical optimization technique for tuning of the PID parameters is also introduced. Illustrative examples are given to demonstrate the performance of the method. Significant improvement is provided in comparison with some previous methods.
\end{abstract}

Index Terms - Time Delay, PID Control, Transient Response Control, Settling Time, Overshoot.

\section{INTRODUCTION}

$\mathrm{S}$ YSTEMS with energy storage cannot respond instantaneously and exhibit transient responses in the time domain whenever they are subjected to inputs or disturbances. In many practical cases, the performance characteristics of the control systems are described in terms of transient response specifications. The most significant specifications are settling time $\left(t_{s}\right)$ and maximum overshoot $\left(M_{p}\right)[1,2]$.

It is a very important specialty for a control method to design the control system providing the target performance specifications. The design of controller which results only the desired transient response is called as transient response control. Although good transient response is one of the most significant requirements for the control system design, there are very few results dealing with the transient response control in the literature [3]. Hauksdóttir [4] pointed out that the analytical expressions of transient responses can be obtained if the poles of the transfer function are all real, negative, and distinct. It was also shown that these formulae in closed form can be used to determine the zeros that result in minimum transient time. Jung et al [5] and Goodwin et al [6] found some limitations on transient response in terms of poles and zeros of the system. An interesting result was also reported by Leon de la Barra [7] who has shown that there are some relations between the nonminimum phase zeros and the case of undershooting [3]. The transient response control studies

S. E. HАMAMCI is with the Department of Electrical-Electronics Engineering Department, Inonu University, Malatya, Turkey (e-mail: serdar.hamamci@inonu.edu.tr).

Copyright (C) BAJECE considered in these methods entirely deal with the relation between the transient response specifications and the poles/zeros of the characteristic polynomial. However, there is a different approach on this topic based on certain relationships between transient response specifications and coefficients of the characteristic polynomial. At first, these relations were introduced by Graham and Lathrop [8] in 1953. They proposed ITAE (Integral Time Absolute Error) standard form method which gives the different forms for each order of characteristic polynomial. This is very inconvenient when the order varies in the course of design [9]. To improve the control system response, a standard form with less oscillation and overshoot was given by Kessler [10]. The control system designed by "Kessler canonical form" is more stable than the ITAE form and has the overshoot of $8 \%$ in the unit step response. In addition to these previous forms, various standard forms such as ITSE (Integral Time Squared Error) form [11] and ISTE (Integral Squared Time Error) form [12] which give more successful time domain responses have been proposed recently. However, it is not possible to design the closed loop systems by these methods according to the transient response specifications which are specified prior to the design. In 1969, Naslin [13] observed empirically that the step response of allpole systems of various orders remains essentially unchanged provided that the coefficients of the characteristic polynomial satisfy certain relationships. Thus, Naslin obtained the explicit formulae between the performance specifications and the coefficients of characteristic polynomial [3, 14]. Using the Naslin's relations, an important contribution in this regard was presented by Manabe [15] who proposed a new control technique, namely Coefficient Diagram Method (CDM), for the linear time-invariant control systems. The most important properties of this method are adaptation of the polynomial representation in the design methodology, use of the twodegrees of freedom (2DOF) control system structure and utilization of Standard Manabe form for obtaining of a unitstep response without overshoot. Furthermore, determination of the desired settling time at the start is another significant feature of the CDM. This is a major advantage which guides to the designer in his design $[15,16]$. For the time delay systems, however, Manabe's method is ineffective to obtain the desired transient response. The obtained transient response does not meet the specified settling time value and nonovershooting property because of the time delay element [16]. As a result of this problem, a modification on the CDM technique is needed. 
In this study, the results of the CDM which has been successfully applied to the linear time-invariant systems are generalized to the case of time delay systems. Since the CDM gives the higher order polynomial controllers whose degrees depend on the degree of the system, a methodology to design the fixed and lowest order controllers as possible such as PID controllers for time delay systems is considered. A new PID tuning method is proposed to achieve the desired settling time and maximum overshoot values which are determined in advance of the design. To achieve this, the first-order plus time delay (FOPTD) model of the stable, integrating or unstable plant is used. Using the 2DOF control system structure, an extra feedforward controller is designed to provide the better closed loop performance focusing on both tracking the desired reference signal and disturbance rejection. This extra controller is tuned in terms of the PID controller. This is an important advantage of the proposed method because the main controller and the extra feedforward controller in other methods are tuned separately [17, 18]. Furthermore, a graphical optimization step is included for modifying the standard Manabe form values to obtain the closed loop response which meets the transient response specifications determined before the design.

\section{PID CONTROLLER DESIGN}

\section{A. 2DOF PID Control System Structure}

A general form of the 2DOF control system is shown in Fig. 1 where $G(s)$ is the plant to be controlled, $C_{f}(s)$ is the feedforward controller and $C(s)$ is the main controller [20]. Successful reference input tracking and the disturbance rejection are provided if

$C(0)=\infty, \quad C_{f}(0) / C(0)=0$

imposes conditions on the controller. The most important result satisfying (1) are that $C(s)$ must contain an integrator and $C_{f}(s)$ must not. Thus, $C(s)$ can be chosen as

$C(s)=K_{p}\left(1+\frac{1}{T_{i} S}+T_{d} s\right)$

in the form of classical PID controller and $C_{f}(\mathrm{~s})$ is a controller satisfying (1).

The polynomial based CDM block diagram [15] is shown in Fig 2. In this figure, $N(s)$ is the numerator and $D(s)$ is the denominator polynomials of the plant transfer function. $B(s)$ and $F(s)$ are the feedback and reference numerator polynomials while $A(s)$ is the forward denominator polynomial

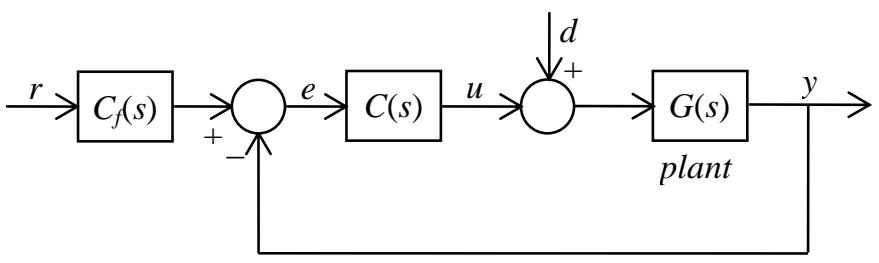

Fig. 1. Two-degrees of freedom control system structure ( $r$ : reference input signal, $y$ : output signal, $u$ : control signal, $d$ : external disturbance signal).

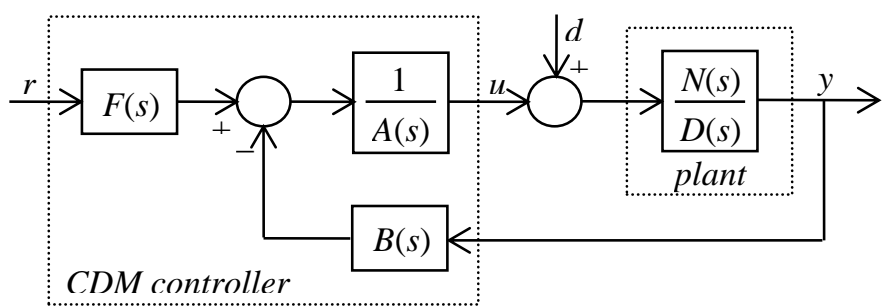

Fig. 2. The block diagram of the CDM control system.

of the controller transfer function. This controller transfer function with two numerators points to a 2DOF system structure. $A(s)$ and $B(s)$ are represented in the polynomial form

$A(s)=\sum_{i=0}^{p} l_{i} s^{i}$ and $B(s)=\sum_{i=0}^{q} k_{i} s^{i}$

where $p$ and $q$ are the orders of the polynomials. The polynomial $F(s)$ is in fact a proportional controller and used to eliminate the steady-state error. The output equation of the control system in Fig. 2 is

$y=\frac{N(s) F(s)}{P(s)} r+\frac{A(s) N(s)}{P(s)} d$.

The characteristic polynomial, $P(s)$ is described by

$P(s)=A(s) D(s)+N(s) B(s)=\sum_{i=0}^{n} a_{i} s^{i}, \quad a_{i}>0$.

where $n$ is the order of $P(s)$.

If the 2DOF control system structure in Fig. 1 is compared with the CDM control system in Fig. 2, it is clearly seen that $N(s)$ and $D(s)$ in Fig. 2 are the polynomials of $G(s)$ in Fig. 1. Similarly, $A(s), B(s)$ and $F(s)$ in the CDM control system structure shown in Fig. 2 are the polynomials of $C_{f}(s)$ and $C(s)$ in Fig. 1. Therefore, $C(s)$ is expressed by $B(s) / A(s)$ and $C_{f}(s)$ have the form of $F(s) / B(s)$. From (1), $B(s) / A(s)$ can be chosen as the PID controller. The controller polynomials then must be chosen as

$A(s)=l_{1} s$,

$B(s)=k_{2} s^{2}+k_{1} s+k_{0}$.

Comparing (2) with (6a) and (6b), the parameters of the PID controller are simply obtained as

$K_{p}=k_{1} / l_{1}, \quad T_{i}=k_{1} / k_{0}$ and $T_{d}=k_{2} / k_{1}$.

\section{B. Modeling}

The most encountered model for the time delay systems is the FOPTD model whose transfer function is given by

$G_{m}(s)=\frac{K}{T_{1} s+T_{0}} e^{-\theta s}$

where $K$ is the gain, $\theta$ is the time delay and $T_{1}$ is the time constant. The value of $T_{0}$ in the model is equal to 1 for the stable processes, 0 for the integrating processes, and -1 for the 
unstable processes. Many experimental identification techniques for the stable, unstable and integrating FOPTD models has been reported in the literature [21-23].

Since the CDM technique considers the transfer function of the plant as the ratio of two independent polynomials, the LTI dynamical model of the plant over a desired operating range should be chosen. Therefore, first order Pade approximation of the term $e^{-\theta s}$

$e^{-\theta s}=(1-0.5 \theta s) /(1+0.5 \theta s)$.

is used. The first order approximation is compulsory to obtain PID controller because their higher number results more complex controllers. The simulation results show that the first order approximation for the time delay gives good results. Substituting (9) into (8), the equivalent LTI model is obtained as

$G_{m}(s)=(-0.5 K \theta s+K) /\left(0.5 T_{1} \theta s^{2}+\left(T_{1}+0.5 T_{0} \theta\right) s+T_{0}\right)$

Remark 2.1: For unstable time delay systems, the condition of $T_{1} \neq 0.5 \theta$ must be fulfilled to not occur pole-zero cancellations in the equivalent LTI model in (10).

\section{Tuning the PID Controller}

From (10), the polynomials of the equivalent LTI model are obtained as

$N(s)=-0.5 K \theta s+K$

$D(s)=0.5 T_{1} \theta s^{2}+\left(T_{1}+0.5 T_{0} \theta\right) s+T_{0}$

Replacing these polynomials and the controller polynomials in (6a-b) into (5), the characteristic polynomial depending on unknown controller parameters $\left(k_{i}\right.$ and $\left.l_{i}\right)$ is obtained. Then, selecting the CDM design parameters a target characteristic polynomial $P_{\text {target }}(s)$ is obtained. According to Manabe [15], the CDM design parameters are stability indices $\left(\gamma_{i}\right)$ and equivalent time constant $(\tau)$ which are specified prior to the design [16].

The equivalent time constant which is defined as a measure of time response speed of the control system in the CDM is given as

$\tau=a_{1} / a_{0}$,

The equivalent time constant is related by the settling time. If $\tau$ is increased, $t_{s}$ is also increased.

The Stability indices indicating the relative stability measure of the control system is described by

$\gamma_{i}=\frac{a_{i}^{2}}{a_{i+1} a_{i-1}}, \quad i=1 \sim(n-1), \quad \gamma_{0}=\gamma_{\infty}=\infty$,

The stability index $\gamma_{1}$ in all indices is much effective on the overshoot. Bigger $\gamma_{1}$ results more relative stability and less overshoot.
For the stability indices, we make use of standard Manabe values in the CDM design. Standard Manabe form [15] is the closed-loop performance measure that gives a unit step response without overshoot and with desired settling time. To achieve this, Manabe proposes the values of the stability indices as follows

$\gamma_{i}=\{2.5,2,2, \ldots \ldots, 2\} \quad i=1 \sim(n-1)$

The choosing of these values gives the unit-step response without overshoot. When the Manabe's standard values are used, the relation between $t_{s}$ and $\tau$ is obtained as

$t_{s} \cong 2.5 \tau$

From (12) and (13), the coefficients $a_{i}$ of $P(s)$ in (5) can be written as

$a_{i}=\tau^{i} a_{0} / \prod_{j=1}^{i-1} \gamma_{i-j}^{j}=Z_{i} a_{0}$.

Thus, the coefficients of the $P(s)$ in (5) can be expressed in terms of $\tau$ and $\gamma_{i}$ as

$P_{\text {target }}(s)=a_{0}\left[\left\{\sum_{i=2}^{n}\left(\prod_{j=1}^{i-1} \frac{1}{\gamma_{i-j}^{j}}\right)(\tau s)^{i}\right\}+\tau s+1\right]$.

Placing the chosen design parameters into $P_{\text {target }}(s)$ above and equating (5) with (17),

$A(s) D(s)+N(s) B(s)=P_{\text {target }}(s)$

is obtained. Assuming $a_{0}=1$ in (17) and solving this equation, the parameters of CDM controller are found as

$l_{1}=\left(\gamma_{1}^{2} \gamma_{2} \theta^{3}+2 \tau \gamma_{1}^{2} \gamma_{2} \theta^{2}+4 \tau^{2} \gamma_{1} \gamma_{2} \theta+8 \tau^{3}\right) /\left[4 \gamma_{1}^{2} \gamma_{2} \theta\left(T_{0} \theta+2 T_{1}\right)\right]$,

$k_{2}=\left(\gamma_{1}^{2} \gamma_{2} T_{1} l_{1} \theta-2 \tau^{3}\right) /\left(\gamma_{1}^{2} \gamma_{2} K \theta\right)$,

$k_{1}=\left(\tau+0.5 \theta-T_{0} l_{1}\right) / K$,

$k_{0}=1 / K$.

Note that the proposed design method needs only two stability indices, $\gamma_{1}$ and $\gamma_{2}$. Finally, the PID controller parameters are obtained from (7).

The numerator polynomial $F(s)$ which is described as a proportional controller is determined to be

$F(s)=P(s) /\left.N(s)\right|_{s=0}=P(0) / N(0)=1 / K$.

In this case, any steady-state error occurring in the time response is reduced to zero. Finally, the feedforward controller in Fig. 1 is turned into

$C_{f}(s)=\frac{F(s)}{B(s)}=\frac{k_{0}}{k_{2} s^{2}+k_{1} s+k_{0}}=\frac{1}{T_{i} T_{d} s^{2}+T_{i} s+1}$. 
Remark 3.2: In this method, the designer does not need to make extra calculations for the feedforward controller. Because the parameters of $C_{f}(s)$ depend on the PID parameters directly as shown in (21).

\section{Graphical Optimization}

The main purpose of the transient response control is to design the controller ensuring the unit step response with desired settling time and maximum overshoot requirements, which are specified prior to design, for the closed loop system. As mentioned in Section 2, using the CDM and standard Manabe form, this purpose can be achieved for the LTI systems easily. However, for the time delay systems, transient response results may not meet the specified performance because of the time delay in the plant. Furthermore, some stability index values which are different from the values of standard Manabe form can give the better performance results. Unfortunately, a certain rule for the selection of the stability indices for the time delay systems has not reported in the literature until now. Therefore, a graphical optimization technique is incorporated to the proposed PID tuning method.

The graphical optimization technique uses two colored figures which are obtained from the colored contour of their 3$\mathrm{D}$ figures. The abscissa of the figures is the first stability index $\left(\gamma_{1}\right)$ and the ordinate is the equivalent time constant $(\tau)$. In the first figure, the maximum overshoot $\left(M_{p}\right)$ values of the closed loop unit step responses calculated by using (7) and (21) versus $\gamma_{1}$ for the interval of $\left(\gamma_{1 \min }, \gamma_{1 \max }\right)$ and $\tau$ for the interval of $\left(\tau_{\min }, \tau_{\max }\right)$ are plotted. Here, $\gamma_{1 \min }, \gamma_{1 \max }, \tau_{\min }, \tau_{\max }$ are the minimum and maximum values of the $\gamma_{1}$ and $\tau$. The internal $\left(\gamma_{1 \min }, \gamma_{1 \max }\right)$ for the first stability index are chosen so that the standard Manabe values are in the middle of this interval. The recommended initial interval is $(2,3)$ and this interval can be modified according to design requirements. The selection of the interval $\left(\tau_{\min }, \tau_{\max }\right)$ depends on the settling time needs of the designer. In the second figure, the settling time $\left(t_{s}\right)$ values of the closed loop unit step responses using (7) and (21) versus $\gamma_{1}$ and $\tau$ are plotted for the same interval in the first figure. These two figures are called as transient response-map (tr-map). Since the proposed method has three design parameters $\left(\gamma_{1}, \gamma_{2}\right.$ and $\left.\tau\right)$ for the FOPTD system, to consider the second stability index $\gamma_{2}$ on the same figures is difficult. However, it is noted that different choices of $\gamma_{2}$ lead to different transient response performances. By changing $\gamma_{2}$ in the range of $(2,3)$ with 0.1 steps, the set of tr-maps is obtained.

The most important advantage of the graphical optimization technique is that the PID controller parameters which are calculated according to the FOPTD model are optimized in consideration of the real plant. Furthermore, an important advantage of this graphical optimization on the mathematical optimization procedures is that the designer can make a decision for the desired performance in a wide performance range visually. It is possible to change the selections of the design parameters according to various transient response alternatives.
In order to show the effectiveness of the graphical optimization, we consider a first-order plus time delay system $G(s)=e^{-1.2 s} /(s+1)$. When $\gamma_{2}=2$ is chosen as in the standard Manabe form, the tr-map of the control system designed by the proposed method is obtained as shown in Fig. 3a. As can be seen from this figure, the standard Manabe form values $\gamma_{i}=\{2.5,2\}$ gives the unit-step response without overshoot and with the shortest settling time at the value of $\tau=3$.3. If $\tau$ is decreased for obtaining the less settling time, the overshoot of the unit step response exceeds the limit of $2 \%$. From Fig. 3a, however, the shorter settling time without overshoot can be obtained at $\gamma_{1}=2.65$ and $\tau=2.5$. Note that the chosen $\gamma_{1}$ is different from the value in the standard Manabe form in this case. Furthermore, the better performances can be obtained by changing $\gamma_{2}$. For the various $\gamma_{2}$ values in the range of $(2,3)$, new tr-maps can be plotted. For example, a tr-map for $\gamma_{2}=2.6$ is shown in Fig. 3b. From this figure, it can be seen that the values $\gamma_{1}=2.5$ and $\tau=2.2$ give the shorter settling time.

For three cases, the unit step responses of the closed loop system are shown in Fig. 4. This figure shows that the last case $\left(\gamma_{i}=\{2.5,2.6\}, \tau=2.2\right)$ gives the shortest settling time. For all cases, the unit step responses are without overshoot.

The presented PID tuning algorithm for stable, unstable or integrating time delay system is summarized as follows:
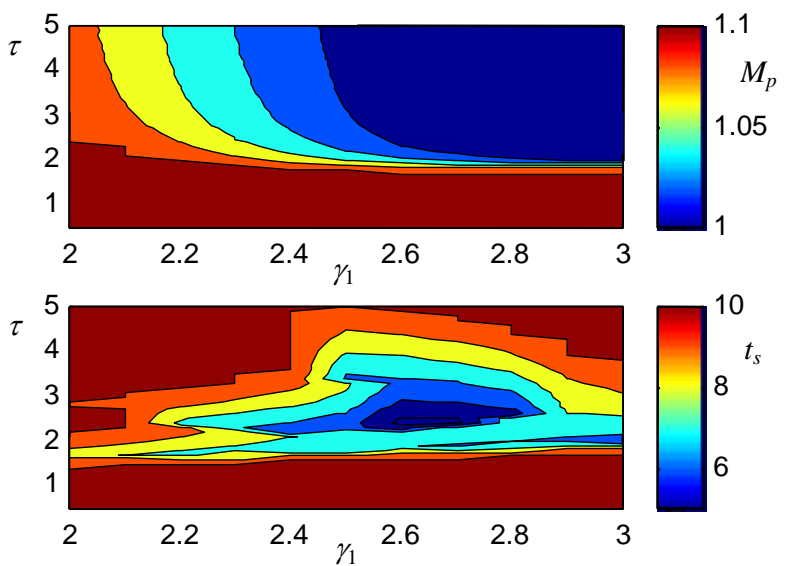

(a)
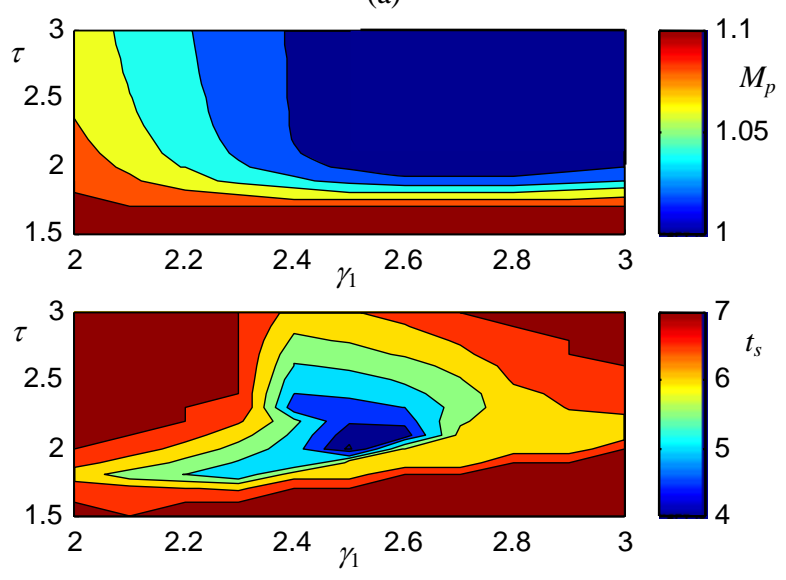

(b)

Fig. 3. The tr-maps for various $\gamma_{2}$ values a) $\gamma_{2}=2$, b) $\gamma_{2}=2.6$. 


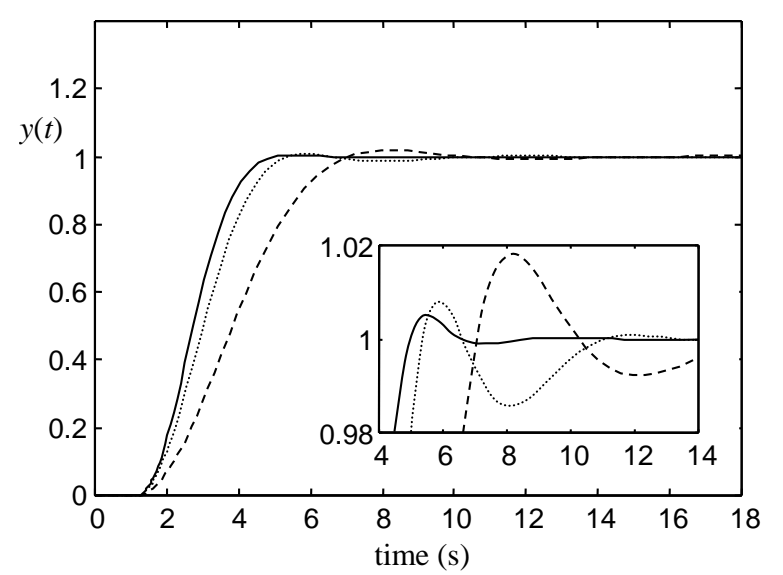

Fig. 4. Unit step responses: (Case 1: $-\gamma_{i}=\{2.52 .6\}, \tau=2.2$; Case 2: $\cdots \cdots \cdots \cdots$ $\gamma_{i}=\{2.652\}, \tau=2.5$; Case 3: $-\cdots \gamma_{i}=\{2.52\}, \tau=3.3$ )

1. Determine a FOPTD model of the time delay system using the methods given in [21-23].

2. Obtain the set of tr-maps by using various values of $\gamma_{2}$ :

a) For a first value of $\gamma_{2}\left(\gamma_{2}=2\right)$, plot the tr-map using (7) and (21) (The abscissa of the tr-map is $\gamma_{1}$ in the range of $\left(\gamma_{1 \text { min }^{-}}\right.$ $\left.\gamma_{1 \max }\right)$ and the ordinate is $\tau$ in the range of $\left(\tau_{\min }-\tau_{\max }\right)$ ).

b) Repeat the same process for the $\gamma_{2}$ values in the range of $(2,3)$ with 0.1 steps.

3. Specify the optimal values of $\gamma_{1}, \gamma_{2}$ and $\tau$.

4. Calculate the PID parameters using $\gamma_{1}, \gamma_{2}$ and $\tau$.

\section{SimULATION EXAMPLES}

In this section, three simulation examples containing systems of different dynamics are given to demonstrate the wide applicability of the introduced PID control method. The first example which considers an unstable FOPDT plant shows the improved performance of the proposed PID tuning method compared to the performances of some methods in the literature. The second example takes into account an integrating FOPDT plant, where it is observed that the proposed method yields significantly improved results relative to recent two PID control methods. In the third example, a higher order system are controlled.

\section{A. Example 1}

Consider a first order unstable system with a time delay $G(s)=4 e^{-2 s} /(4 s-1)$. The PID controller parameters calculated by ISTE optimization method of Visioli [24] are $K_{p}=0.652, T_{i}=8.261, T_{d}=0.9671$ and Taylor series expansion method of Sree et al [25] are $K_{p}=0.548, T_{i}=11.117, T_{d}=1.024$. Simulation results show that the best settling time for these PID control systems is about $25 \mathrm{~s}$. Therefore, a smaller settling time than this value is aimed to obtain the better performance for the proposed method. First of all, a set of tr-maps computed for the various values of $\gamma_{2}$ in the range of $(2,3)$ are plotted. From these tr-maps, the choice of $\gamma_{2}=2.2$ gives the shortest settling time without overshoot as shown in Fig. 5. Also seen from this figure that $\tau=5.7$ and $\gamma_{1}=2.4$ values for $\gamma_{2}=2.2$ is the best selection. Therefore, the PID controller parameters are calculated as $K_{p}=0.5384, \quad T_{i}=12.5086$,
$T_{d}=0.6891$ and the feedforward controller is obtained as $C_{f}(s)=1 /\left(8.6197 s^{2}+12.509 s+1\right)$.

The unit step responses of the proposed PID control system and two PID control systems designed by Visioli and Sree $e t$ $a l$ are shown in Fig. 6a while the control signals produced by these control systems are shown in Fig. 6b. It is apparent that the proposed PID controller produces a unit step response without an overshoot and with the smallest settling time, and this is achieved by a control signal having a smaller magnitude.
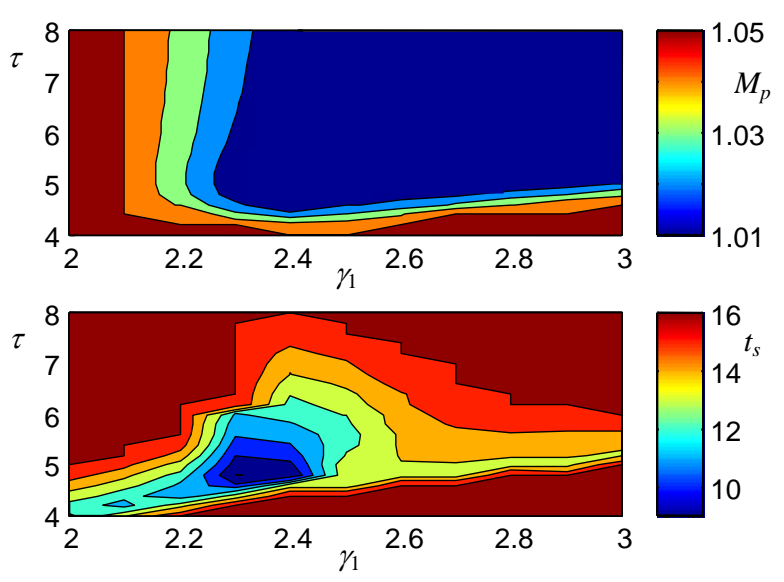

Fig. 5. The optimal tr-map for Example 1.

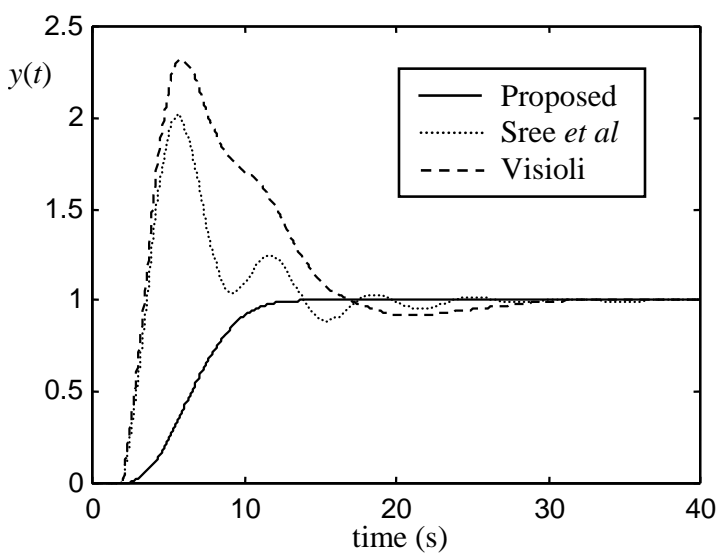

(a)

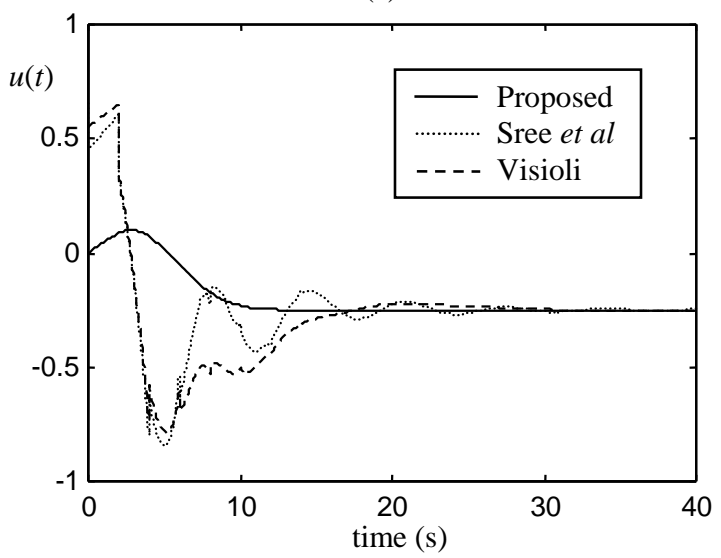

(b)

Fig. 6. a) Unit step responses, b) Control signals (Example 1). 


\section{B. Example 2}

An integrating plant transfer function $G(s)=e^{-s} / 5 s$ is considered in this example. The PID controller parameters for ISTE optimization method of Visioli [24] are obtained as $K_{p}=6.7, T_{i}=1.83, T_{d}=0.49$ and for the frequency response method of Wang and Cluett [26] are calculated as $K_{p}=4.794$, $T_{i}=3.043, T_{d}=0.391$. The Wang and Cluett's method gives the best settling time which is $7.5 \mathrm{~s}$.

For the proposed method, the optimal tr-map which is obtained for $\gamma_{2}=2.3$ is shown in Fig. 7. In accordance with this
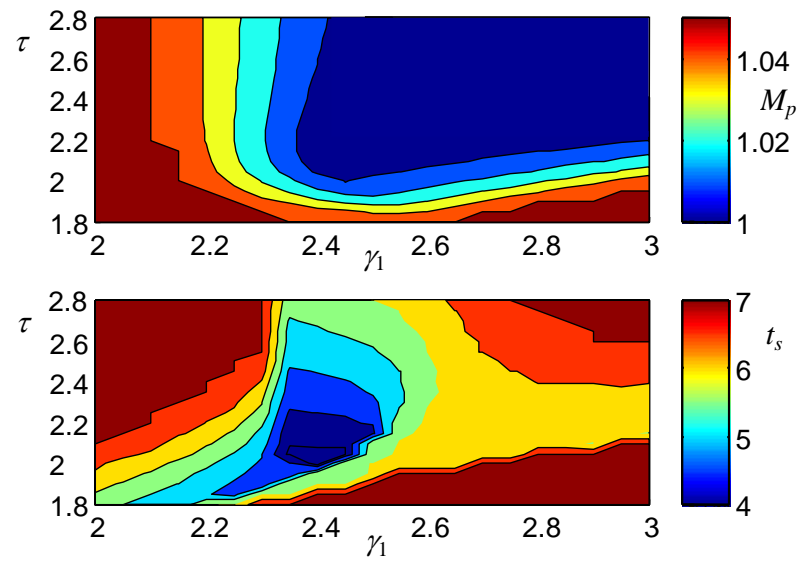

Fig. 7. The optimal tr-map for Example 2.

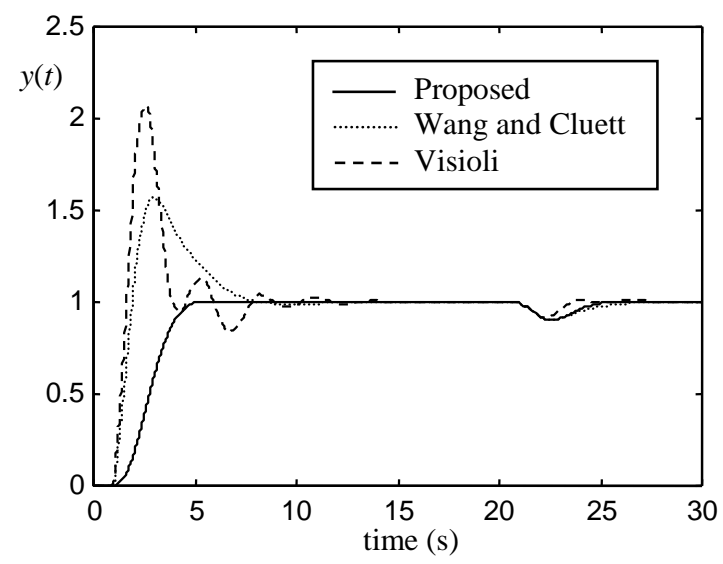

(a)

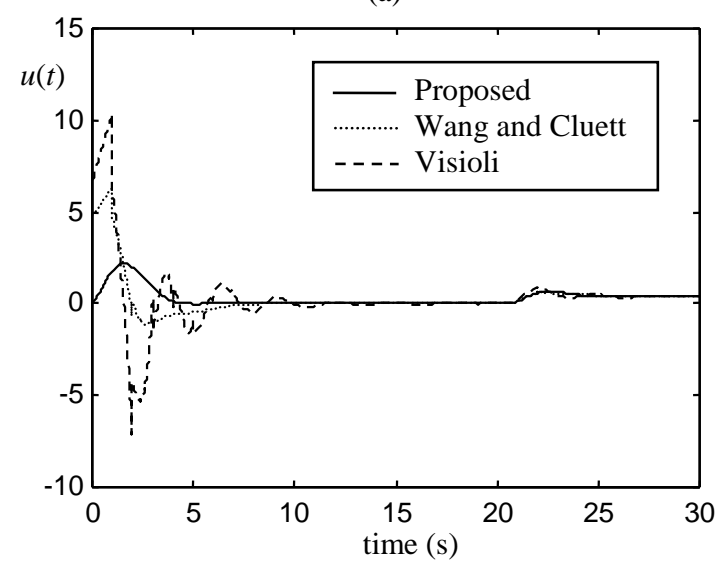

(b)

Fig. 8. a) Unit step responses, b) Control signals (Example 2). map, the PID controller parameters obtained by using the graphical optimization method with the design parameters $\tau=2.3$ and $\gamma_{i}=\{2.4,2.3\}$ are $K_{p}=5.1461, T_{i}=2.8$ and $T_{d}=0.3156$. The feedforward controller is $C_{f}(s)=1 /\left(0.8837 s^{2}+2.8 s+1\right)$.

The unit step response of the proposed PID control system is compared with those of control systems using the previous PID controllers are shown in Fig. 8a for a step loaddisturbance change with magnitude of $d=-0.5$ applied at time $20 \mathrm{~s}$. From this figure, the proposed design method results in an improved performance with shorter settling time and without overshoot. Fig. 8b illustrates the control signals, which show that the introduced design method requires less effort for the improved control action, for all PID methods.

\section{Example 3}

A high order plant transfer function of $G(s)=1 /(s+1)^{8}$, which was given in Wang and Shao [29], is taken into account in this example. The FOPDT model for this plant was given as $G(s)=e^{-5.1 s} /(4.35 s+1)$ in [27]. The PID parameters were determined as $K_{p}=0.677, T_{i}=4.3314$ and $T_{d}=1.6489$ using the method of Wang and Shao and estimated as $K_{p}=0.716$, $T_{i}=5.4589$ and $T_{d}=1.3380$ through the method of Cluett and Wang [28]. For the improved performance, the design parameters of the proposed method are chosen as $\tau=8.4$ and $\gamma_{i}=\{2.05,4.2\}$ for the optimal tr-map shown in Fig. 11. The PID parameters with these values are calculated as $K_{p}=1.0012$,
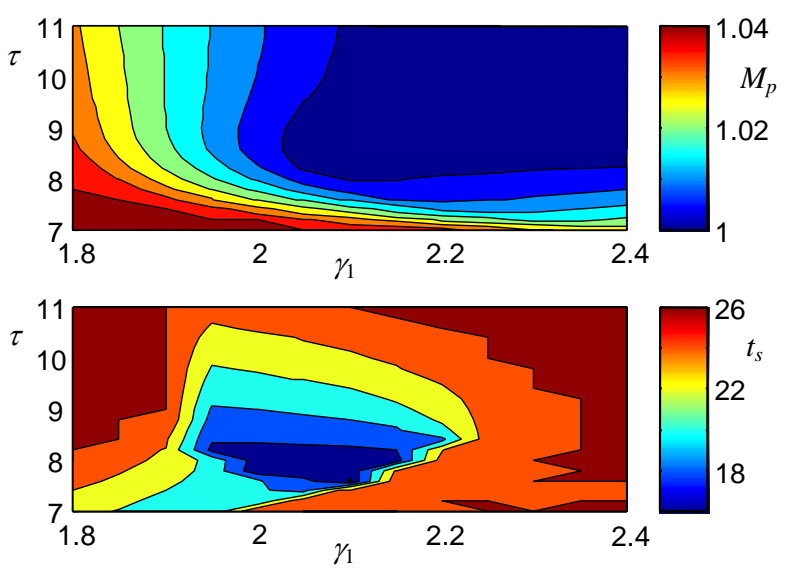

Fig. 11. The optimal tr-map for Example 3.

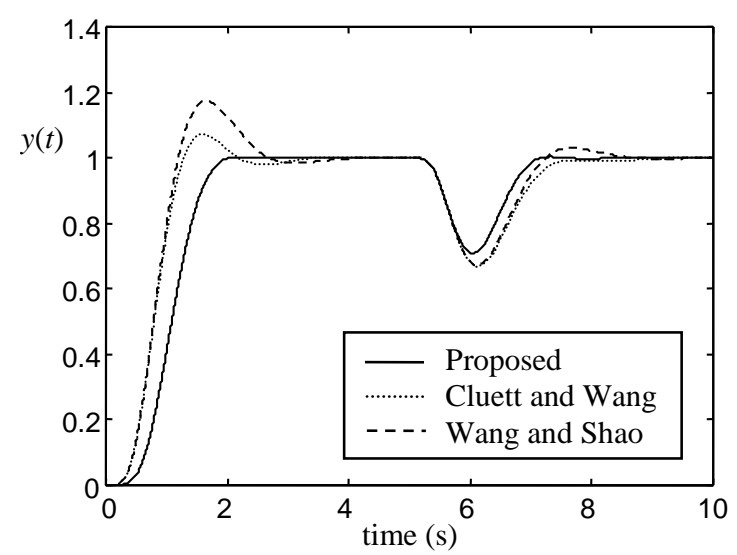

Fig. 12. Unit step responses (Example 3). 
$T_{i}=5.4782$ and $T_{d}=1.9411$ and the feedforward controller is obtained as $C_{f}(s)=1 /\left(10.6336 s^{2}+5.4782 s+1\right)$. The outputs to a unit step input and step load disturbance of magnitude 0.5 introduced at time 50s. are shown in Fig. 12 for all the design studies. It can be shown from this figure that the proposed method provides excellent control for the high-order plant in terms of settling time and overshoot.

\section{CONCLUSIONS}

In this paper, the earlier results on transient response control which was successfully applied to the control of linear timeinvariant systems using higher order polynomial controllers have been extended to the case of the control of linear time delay systems using PID controllers. The solution to the problem of PID transient response control presented here is based on first obtaining the explicit PID tuning formulae using the FOPTD model of the plant. To achieve this, the design parameters which are called as the stability indices and the equivalent time constant are considered. Then, the optimal PID controller is obtained by the graphical optimization technique which makes use of transient responses maps (trmaps). The dominant merit of the proposed method is that the numerical quantities of the desired settling time and maximum overshoot values for the unit step response of the closed-loop system are specified before starting the design. As evidenced by the simulation results, it can be concluded that the proposed method gives reliable and accurate results for the stable, integrating and unstable time delay systems.

\section{REFERENCES}

[1] K. Ogata, Modern Control Engineering, Prentice-Hall, Englewood Cliffs, NJ, 1970.

[2] J. Zheng, P. Guo, and J.D. Wang, "STFC-self-tuning fuzzy controller," in Proc. IEEE Conf. on Systems, Man and Cybernetics, Chicago, 1992.

[3] Y.C. Kim, L.H. Keel, and S.P. Bhattacharyya, "Transient response control via characteristic ratio assignment," IEEE Trans. on Automatic Control, vol. 48, pp. 2238-2244, 2003.

[4] A.S. Hauksdóttir, "Analytic expression of transfer function responses and choice of numerator coefficients (Zeros)," IEEE Trans. on Automatic Control, vol. 41, pp. 1482-1488, 1996.

[5] S. Jung, and R.C. Dorf, "Novel analytic technique for PID and PIDA controller design," in Proc. $13^{\text {th }}$ IFAC World Congress, San Francisco, USA, 1996.

[6] G.C. Goodwin, A.R. Woodyatt, R.H. Middleton, and J. Shim, "Fundamental limitations due to $j w$-axis zeros in SISO systems," Automatica, vol. 35, pp. 857-863, 1999.

[7] B.A. Leon de la Barra, "On undershoot in SISO systems," IEEE Trans. on Automatic Control, vol. 39, pp. 578-581, 1994.

[8] D. Graham, and R.C. Lathrop, "The synthesis of "optimum" transient response: criteria and standard forms," AIEE Transactions, vol. 72, pp. 273-288, 1953

[9] G.F. Franklin, and J.D. Powell, and A. Emami-Naeini, "Feedback control of dynamic systems," Addison-Wesley, 1994.

[10] C. Kessler, "Ein beitrag zur theorie mehrschleifiger regelungen," Regelungstechnik, vol. 8, pp. 261-266, 1960.
[11] N.K. Sinha, Control Systems, $2^{\text {nd }}$ ed. John Wiley \& Sons Inc, 1994.

[12] M. Zhuang, and D.P. Atherton, "Automatic tuning of optimum PID controllers," IEE Proc. D, vol. 140, pp. 216-224, 1993.

[13] P. Naslin, Essentials of Optimal Control, Boston Technical Publishers, Massachusetts, 1969.

[14] Y.C. Kim, L.H. Keel, and S.P. Bhattacharyya, "Transient response control via characteristic ratio assignment and pulsatance assignment," in Proc. $21^{\text {th }}$ American Control Conference, Anchorage, 2002.

[15] S. Manabe, "Coefficient diagram method," in Proc. 14 $4^{\text {th }}$ IFAC Symposium on Automatic Control in Aerospace, Seoul, 1998.

[16] S.E. Hamamci, "A robust polynomial-based control for stable processes with time delay," Electrical Engineering, vol. 87, pp. 163-172, 2005.

[17] T. Liu, W. Zhang, and D. Gu, "Analytical design of two-degree-offreedom control scheme for open-loop unstable processes with time delay," Journal of Process Control, vol. 15, pp. 559-572, 2005.

[18] A.S. Rao, and M. Chidambaram, "Enhanced two-degrees-of-freedom control strategy for second-order unstable processes with time delay," Ind. Eng. Chem. Res., vol. 45, pp. 3604-3614, 2006.

[19] S. Manabe, and Y.C. Kim, "Recent development of Coefficient Diagram Method," in Proc. $3^{\text {rd }}$ Asian Control Conference, Shanghai, 2000.

[20] M. Araki, and H. Taguchi, "Two-degree-of-freedom PID controllers," Int. J. of Control, Automation, and Systems, vol. 1, pp. 401-411, 2003.

[21] I. Kaya, and D.P. Atherton, "Exact parameter estimation from relay autotuning under static load disturbances," in Proc. $20^{\text {th }}$ American Control Conference, Arlington, 2001.

[22] A. Visioli, "Time-optimal plug\&control for integrating and FOPDT processes," Journal of Process Control, vol. 13, pp. 195-202, 2003.

[23] S. Majhi, and D.P. Atherton, "Online tuning of controllers for an unstable FOPDT process," IEE Proc.- Control Theory Appl., vol. 147, pp. 421-427, 2000.

[24] A. Visioli, "Optimal tuning of PID controllers for integral and unstable processes," IEE Proc.-Control Theory Appl., vol. 148, pp. 180-184, 2001.

[25] R.P. Sree, M.N. Srinivas, and M. Chidambaram, "A simple method of tuning PID controllers for stable and unstable FOPDT systems," Comput. Chem. Eng., vol. 28, pp. 2201-2218, 2004.

[26] L. Wang, and W.R. Cluett, "Tuning PID controllers for integrating processes," IEE Proc.- Control Theory Appl., vol. 144, pp. 385-392, 1997.

[27] I. Kaya, "Tuning PI controllers for stable processes with specifications on gain and phase margins," ISA Transactions, vol. 43, pp. 297-304, 2004.

[28] W.R. Cluett, and L. Wang, "New tuning rules for PID control," Pulp and Paper Canada, vol. 3, pp. 52-55, 1997.

[29] Y.G. Wang, and H.H. Shao, "PID autotuner based on gain- and phasemargin specifications," Ind. Eng. Chem. Res., vol. 38, pp. 3007-3012, 1998.

\section{BIOGRAPHY}

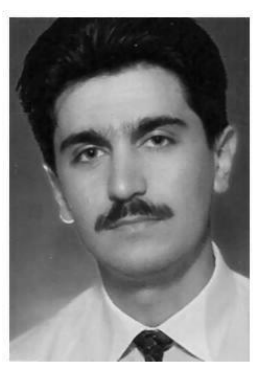

SERDAR E. HAMAMCI received the B.S. degree in electronics engineering from Erciyes University in 1992 and the M.S. and Ph.D. degrees in electrical-electronics enginnering from Firat University in 1997 and 2002 respectively.

From 1993 to 2009, he was a Research Assistant with the Electrical-Electronics Engineering Department at Inonu University. Since 2009, he has been an Associate Professor with the same department. His research interest includes control system design, stabilization and fractional order systems. 